\title{
Nondestructive analysis of single crystals of selenide spinels by X-ray spectrometry techniques
}

\author{
E. Malicka • R. Sitko • B. Zawisza • J. Heimann • \\ D. Kajewski • A. Kita
}

Received: 13 July 2010 /Revised: 20 September 2010 / Accepted: 21 September 2010 /Published online: 6 October 2010

(C) The Author(s) 2010. This article is published with open access at Springerlink.com

\begin{abstract}
The paper presents possibilities and difficulties in nondestructive analysis of small multielement single crystals performed by means of X-ray spectrometry techniques: micro-X-ray fluorescence spectrometry $(\mu-X R F)$, energydispersive electron probe microanalysis (ED-EPMA), and $\mathrm{X}$-ray photoelectron spectroscopy (XPS). The capability of the X-ray spectroscopy techniques in elemental analysis is demonstrated with the single crystals of selenide spinels of the general formula $\mathrm{M}_{x} \mathrm{~N}_{y} \mathrm{Cr}_{z} \mathrm{Se}_{4}\left(\mathrm{M}^{+2}\right.$ and $\mathrm{N}^{+3}$ are, for example, $\mathrm{Zn}^{+2}, \mathrm{~V}^{+3}, \mathrm{Ga}^{+3}, \mathrm{Cd}^{+2}, \mathrm{In}^{+3}$, and $\mathrm{Sb}^{+3}$ ). The results of the nondestructive analyses ( $\mu$-XRF, ED-EPMA, and XPS) are compared with those obtained by inductively coupled plasma optical emission spectrometry (ICP-OES) and wavelength-dispersive X-ray spectrometry (WDXRF) following sample digestion. The present study shows satisfactory agreement between the results of $\mu$-XRF analysis performed using the standardless fundamental parameter method and the results obtained with the WDXRF and ICP-OES analyses. If the measured single crystal is precisely positioned, the difference between $\mu$ XRF and wet analysis (WDXRF and ICP-OES) does not
\end{abstract}

Published in the special issue Focus on Analytical Science in Poland (VIII Polish Conference on Analytical Chemistry) with Guest Editor Pawel Koscielniak

E. Malicka $(\square) \cdot$ R. Sitko $\cdot$ B. Zawisza $\cdot$ A. Kita

Institute of Chemistry, University of Silesia,

Szkolna 9,

40-006 Katowice, Poland

e-mail: ewa.malicka@us.edu.pl

J. Heimann · D. Kajewski

Institute of Physics, University of Silesia,

Uniwersytecka 4,

40-007 Katowice, Poland exceed 5\% rel. The reliable results of ED-EPMA can be obtained only if the measured area is sufficiently large, i.e., of $200 \times 300 \mu \mathrm{m}$. Even if this condition is fulfilled, the relative difference between the ED-EPMA and the wet analysis may reach $10 \%$ rel. In case of the XPS analysis, the accuracy of results depends on the proper preparation of the sample surface. It should be free of contamination that can be obtained by scraping in situ in ultrahigh vacuum. The ion etching, commonly used for cleaning the surface, leads to preferential sputtering; therefore, the reliable results cannot be obtained.

Keywords Micro-XRF EPMA - XPS · Selenide spinel · Nondestructive analysis $\cdot$ Chalcogenide compounds

\section{Introduction}

The chromium selenospinels are well-known ferromagnetic and antiferromagnetic semiconductors with unique electrical properties [1-4]. The general chemical formula of the compounds can be written as $(\mathrm{A})\left[\mathrm{Cr}_{2}\right] \mathrm{Se}_{4}$, where $\mathrm{A}^{2+}$ represents $\mathrm{Cd}^{2+}, \mathrm{Zn}^{2+}, \mathrm{Cu}^{2+}$, and $\mathrm{Co}^{2+}$ cations. The single crystals of the spinels are grown by a chemical vapour transport method with anhydrous chromium chloride as a transporting agent. This process leads to a growth of the single crystals of dimensions from approximately 0.5 to $5 \mathrm{~mm}$ (Fig. 1) and containing various amounts of the doped elements. Since chemical composition strongly influences electric and magnetic properties of these materials [1-3], detailed information on their stoichiometry is required. The chemical composition can be determined after sample digestion using, for example, inductively coupled plasma optical emission spectrometry (ICP-OES) [4] or 


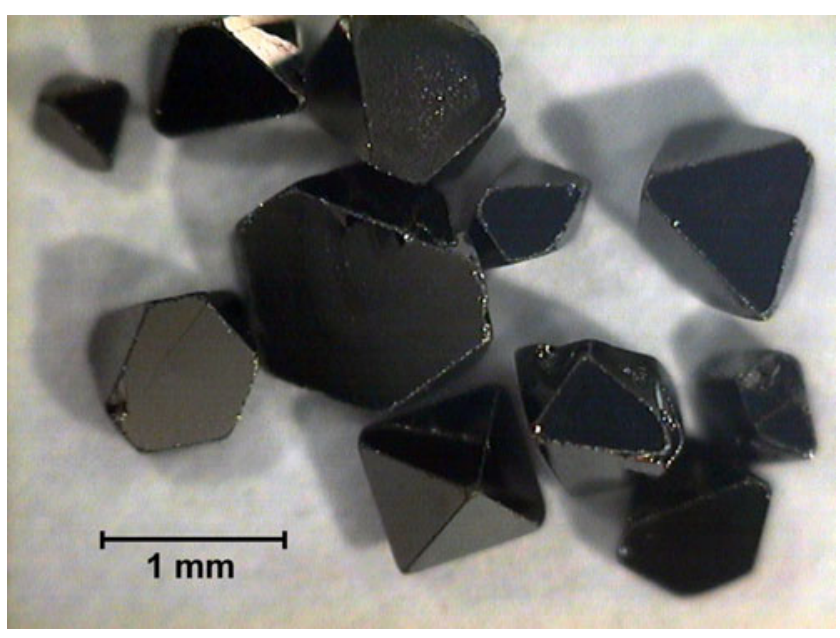

Fig. 1 The single crystals of $\mathrm{Zn}_{1-x} \mathrm{Cd}_{x} \mathrm{Cr}_{2} \mathrm{Se}_{4}$ spinels

wavelength-dispersive X-ray fluorescence spectrometry (WDXRF) [5, 6]. However, the nondestructive methods are much more desirable because investigation of the physical properties is usually carried out for the materials in crystalline state. X-ray fluorescence techniques are ideal tools for analysis of advanced materials of both bulk and thin layers [7-9]. Recently, the nondestructive analysis of monocrystals and polycrystals by using a laboratoryconstructed energy-dispersive X-ray fluorescence spectrometry with monochromated primary radiation has been described [10].

In the present paper, the application of other nondestructive X-ray spectroscopy methods for elemental analysis of the chromium selenospinels of the general formula $\mathrm{M}_{x} \mathrm{~N}_{y} \mathrm{Cr}_{z} \mathrm{Se}_{4}$ (where $\mathrm{M}^{2+}$ and $\mathrm{N}^{3+}$ are $\mathrm{Zn}^{2+}, \mathrm{V}^{3+}, \mathrm{Ga}^{3+}$, $\mathrm{Cd}^{2+}, \mathrm{In}^{3+}$, and $\left.\mathrm{Sb}^{3+}\right)$ is discussed. The synthesized crystals of various compositions were studied by micro-X-ray fluorescence spectrometry ( $\mu$-XRF), energy-dispersive electron probe microanalysis (ED-EPMA), and X-ray photoelectron spectroscopy (XPS). The results of the nondestructive techniques are compared with those obtained from ICP-OES and WDXRF, both requiring the digestion of specimen.

\section{Experimental}

$\mu-X R F$ The single crystals of the spinels of dimensions from approximately 1 to $3 \mathrm{~mm}$ were analyzed without any previous treatment. The measurements were performed with the laboratory-constructed spectrometer described in details in [11]. The samples were excited by an X-ray beam from the air-cooled side-window Rh target of the X-ray tube of $125-\mu \mathrm{m}$ thickness Be window and approximately 100- $\mu \mathrm{m}$ nominal focal spot size (XTF 5011/75, Oxford Instruments, USA). The X-ray tube was supplied with XLG high-voltage generator (Spellman, USA). The size of the analyzed area may be optimized with the tungsten pinhole collimators of the diameter of 100,200,400,1,000, and $2,000 \mu \mathrm{m}$, ensuring the focal spot sizes of $169,280,581$, 1,469 , and $2,888 \mu \mathrm{m}$, respectively. The X-ray spectra emitted by the sample were collected by a thermoelectrically cooled Si-PIN detector (XR-100CR Amptek, Bedford, MA, USA) of $6-\mathrm{mm}^{2}$ active area, $500-\mu \mathrm{m}$ crystal thickness, and $12.5-\mu \mathrm{m} \mathrm{Be}$ window thickness. The resolution of the Si-PIN detector cooled to the temperature of approximately $-55{ }^{\circ} \mathrm{C}$ was $145 \mathrm{eV}$ at $5.9 \mathrm{keV}$. The detector was coupled to a multichannel analyzer (PX4 Amptek, Bedford, MA, USA). The sample position was adjusted by the $\mathrm{X}-\mathrm{Y}$ stage and monitored with the $\mathrm{CCD}$ camera and two laser pointers.

EPMA Microanalysis was performed with EDS analyzer (ISIS-300, Oxford Instruments) coupled to the scanning
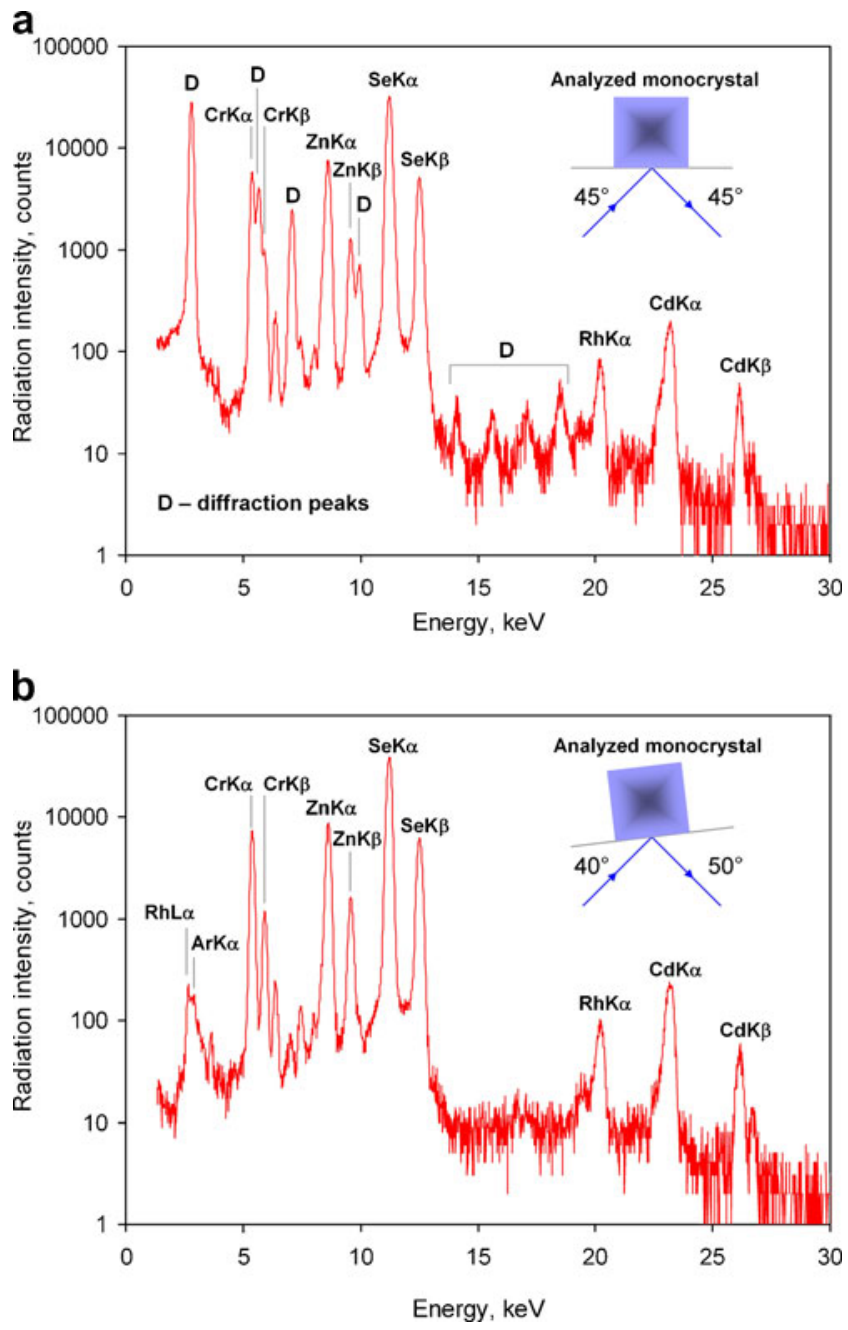

Fig. $2 \mathrm{X}$-ray spectra of $\mathrm{Zn}_{1-x} \mathrm{Cd}_{x} \mathrm{Cr}_{2} \mathrm{Se}_{4}$ collected at various positions of crystal. The high-intensity diffraction peaks $(D)$ can be observed if the incidence and takeoff angles are equal $45^{\circ}$ 
electron microscope (JSM-5410, JEOL). The accelerating voltage was $20 \mathrm{kV}$. The takeoff angle was $45^{\circ}$. The X-ray spectra were collected by liquid-nitrogen-cooled $\mathrm{Si}(\mathrm{Li})$ detector with energy resolution of $130 \mathrm{eV}$. The quantitative analysis was carried out using SEMQuant software (Oxford Instruments) and pure element standards from Agar Scientific.

XPS The XPS spectra were obtained with monochromated Al $\mathrm{K} \alpha$ radiation using PHI 5700/660 Physical Electronic spectrometer. Its hemispherical analyzer ensures resolution of about $0.3 \mathrm{eV}$ in kinetic energy of photoelectrons. Two different modes of purifying the sample from contaminations were applied and compared. In the first run of the experiment, the surface of the sample was scrapped in situ at $10^{-10}-\mathrm{hPa}$ vacuum. In the second run, the profiles were measured by sputtering the outer layer of the sample with contaminations at $1 \mathrm{kV}$ with $\mathrm{Ar}^{+}$.

The following spectra were measured: (1) an overview of the binding energy in the range -2 to $1,400 \mathrm{eV}$; (2) the high-resolution spectrum of the valence band in the region $-2 \div 20 \mathrm{eV}$; and (3) the core-level characteristic peaks for $\mathrm{Se}$ $3 d, \mathrm{Cr}, \mathrm{Cd}$, and $\mathrm{Zn} 2 p_{3 / 2}$. The accurate chemical composition was determined from the core-level characteristic peaks. The background was subtracted using Tougaard's approximation.

WDXRF The single crystal was weighed on a microanalytical balance (the masses of analyzed samples were usually between 5 and $20 \mathrm{mg}$ ). Then the crystal was digested in a $20-\mathrm{mL}$ baker in aqua regia $(1.5 \mathrm{~mL}$ of concentrated $\mathrm{HCl}$ and $0.5 \mathrm{~mL}$ of concentrated $\mathrm{HNO}_{3}$ ). After $20 \mathrm{~min}$ of heating, the material became completely dissolved. The solution was transferred into a $10-\mathrm{mL}$ volumetric flask and filled up to the mark with water. Next, $0.5 \mathrm{~mL}$ of the solution was pipetted onto the Whatman filter $(21 \mathrm{~mm}$ in diameter) and dried under IR heater. The standard samples were prepared by pipetting appropriate solutions of analytes onto the Whatman filters. The prepared samples were analyzed using a wavelength-dispersive X-ray spectrometer with $\operatorname{LiF}(200)$ analyzing crystal and the silver target X-ray tube operating at $45 \mathrm{kV}$ and $40 \mathrm{~mA}$.

ICP-OES The single crystals were digested in the same way as for the WDXRF measurements. The obtained solutions were measured using a sequential spectrometer

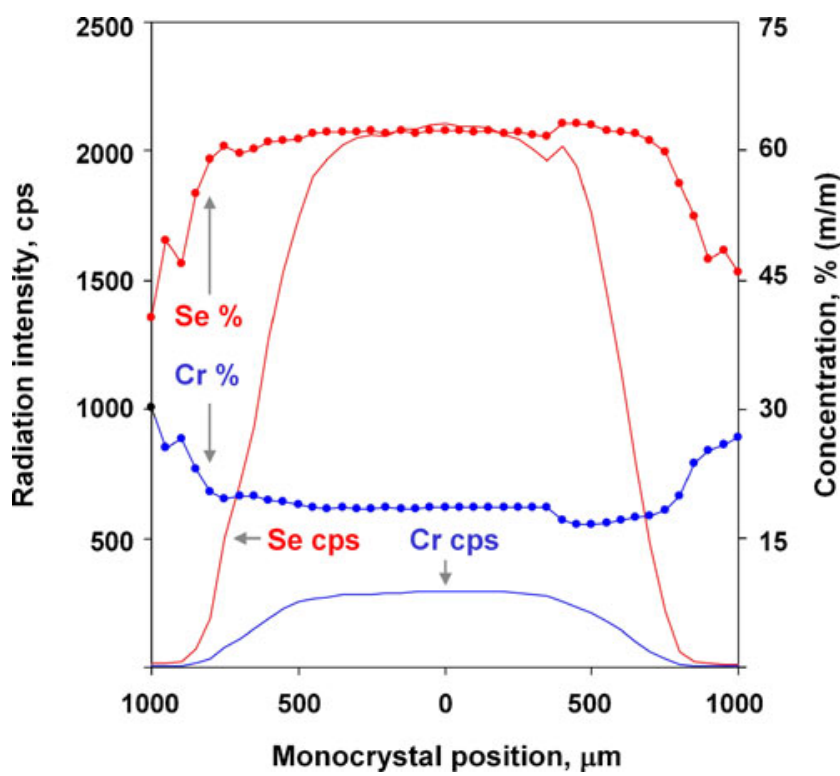

Fig. 3 The intensity of the fluorescent radiation of $\mathrm{Cr}$ and $\mathrm{Se}$ versus the position of the $\mathrm{Zn}_{0.81} \mathrm{Cd}_{0.20} \mathrm{Cr}_{1.99} \mathrm{Se}_{4}$ crystal. The crystal size is approximately $900 \mu \mathrm{m}$ and the pinhole collimator $200 \mu \mathrm{m}$

with an excitation in the ICP plasma made by a SPECTRO Analytical Instruments (Germany) with the following parameters: frequency $27.12 \mathrm{MHz}$, power $1.1 \mathrm{~kW}$, torch $\mathrm{Ar} / \mathrm{Ar} / \mathrm{Ar}$ (quartz, demountable), coolant gas $14.0 \mathrm{Lmin}^{-1}$, auxiliary gas $0.5 \mathrm{Lmin}^{-1}$, nebulizer gas $1.0 \mathrm{Lmin}^{-1}$, nebulizer concentric Meinhard, nebulizer pressure 2.4 bar, spray chamber of glass, according to Scott, sample rate $1.0 \mathrm{mLmin}^{-1}$, observation height $11 \mathrm{~mm}$, holographic grating 2,400 grooves per milliliter, dispersion of grating in first reciprocal order $0.55 \mathrm{nmmm}^{-1}$, and wavelength range of monochromator $165-460 \mathrm{~nm}$.

\section{Results and discussion}

The $\mu$-XRF spectrometry is a valuable tool for the nondestructive multielemental analysis of small specimens. Moreover, this low-cost and non-time-consuming technique can successfully be applied in the screening analysis. Therefore, $\mu$-XRF spectrometry is an ideal tool for the analysis of single crystals of selenide spinels synthesized by chemical vapour transport method. The chemical vapour transport process leads to a growth of a few dozen
Table 1 EDXRF analysis for the $\mathrm{Zn}_{1-x} \mathrm{Cd}_{x} \mathrm{Cr}_{2} \mathrm{Se}_{4}$ singlecrystal set in various positions

Measurements were done using a 200- $\mu \mathrm{m}$ collimator. Results are in percent $(\mathrm{m} / \mathrm{m})$

\begin{tabular}{lllll}
\hline & $\mathrm{Zn}$ & $\mathrm{Cd}$ & $\mathrm{Cr}$ & $\mathrm{Se}$ \\
\hline EDXRF $45^{\circ} / 45^{\circ}$ & $11.29 \pm 0.08$ & $4.5 \pm 0.1$ & $20.6 \pm 0.2$ & $63.6 \pm 0.2$ \\
EDXRF $40^{\circ} / 50^{\circ}$ & $10.73 \pm 0.08$ & $4.5 \pm 0.1$ & $20.9 \pm 0.2$ & $63.8 \pm 0.2$ \\
Rel. difference (\%) & 5.2 & 0.22 & 1.8 & 0.28 \\
\hline
\end{tabular}


Table 2 EDXRF analysis for $\mathrm{Zn}_{0.81} \mathrm{Cd}_{0.20} \mathrm{Cr}_{1.99} \mathrm{Se}_{4}$ crystal in the center and in the position $\pm 600 \mu \mathrm{m}$ off-center

\begin{tabular}{|c|c|c|c|c|c|c|c|}
\hline \multirow[t]{3}{*}{ Element } & \multirow{3}{*}{$\begin{array}{l}\text { In center } \\
\%(\mathrm{~m} / \mathrm{m})\end{array}$} & \multicolumn{2}{|c|}{$+600 \mu \mathrm{m}$ off-center } & \multicolumn{2}{|c|}{$-600 \mu \mathrm{m}$ off-center } & \multicolumn{2}{|c|}{ In the center } \\
\hline & & \multicolumn{2}{|c|}{$(280 \mu \mathrm{m}$ focal spot size $)$} & \multicolumn{2}{|c|}{$(280 \mu \mathrm{m}$ focal spot size $)$} & \multicolumn{2}{|c|}{$(2,888 \mu \mathrm{m}$ focal spot size $)$} \\
\hline & & $\%(m / m)$ & Relative difference $(\%)$ & $\%(\mathrm{~m} / \mathrm{m})$ & Relative difference (\%) & $\%(m / m)$ & Relative difference $(\%)$ \\
\hline $\mathrm{Zn}$ & 10.73 & 10.9 & 1.9 & 10.8 & 0.9 & 10.8 & 0.9 \\
\hline $\mathrm{Cd}$ & 4.5 & 4.5 & 0 & 6.2 & 38 & 4.9 & 8.9 \\
\hline $\mathrm{Cr}$ & 20.9 & 21.6 & 3.3 & 18.9 & -9.6 & 20.4 & -2.4 \\
\hline $\mathrm{Se}$ & 63.8 & 63.0 & -1.3 & 64.1 & 0.5 & 63.9 & 0.2 \\
\hline
\end{tabular}

monocrystals of sizes from approximately 0.5 to $5 \mathrm{~mm}$. Moreover, each monocrystal can be doped with various amounts of element to be interested.

The quantitative $\mu$-XRF analysis was performed using the standardless fundamental parameter (FP) method based on the Sherman equation [12] and Pella et al. algorithm [13, 14] to calculate the X-ray tube spectrum. Our previously reported experiments [11] have shown that the applied pinhole collimators did not modify the spectral distribution of the X-ray tube that is important in FP methods, especially in the standardless analysis. Although $\mu$-XRF spectrometry has many advantages, the accurate results for the single crystals can be obtained if several experimental requirements are met. To these belongs the crystal sample positioning in order to avoid an appearance of strong reflections of the Bragg diffraction. Figure 2 presents the $\mathrm{X}$ ray spectra for $\mathrm{Zn}_{1-x} \mathrm{Cd}_{x} \mathrm{Cr}_{2} \mathrm{Se}_{4}$ collected at various crystal positions. The high-intensity diffraction peaks can be observed if the incidence and takeoff angles are equal $45^{\circ}$. Two problems arise: the diffraction peaks can be misidentificated and first of all the calculated net intensities of fluorescent radiation can be overestimated or underestimated after deconvolution of the spectrum. If the analyzed crystal takes such an orientation that the incidence and takeoff angles are equal $40^{\circ}$ and $50^{\circ}$, respectively, the diffraction peaks may be eliminated, as shown in Fig. 2. Thus, the question is whether the crystal orientation (and diffraction peaks) affects the quantitative analysis. The results of the $\mu$-XRF measurements carried out for the crystal set in various positions are given in Table 1. They show that the diffraction peaks have influence on the quantitative analysis; especially, the $\mathrm{Zn}$ concentrations differ rather significantly. It should be emphasized that the results are normalized during the calculations; therefore, the determined element concentrations are strongly correlated.

The diffraction peaks are not the only source of the potential error in the analysis. High-quality results from the standardless FP method can be obtained if the assumed geometry of measurements (incidence and takeoff angles) is assured. Fortunately, the synthesized crystals have wellformed flat faces that enables easy sample adjusting. Nevertheless, various positions of the sample may strongly influence the quantitative results of the $\mu$-XRF analysis; therefore, this effect was checked in detail. Figure 3 shows the relationship between the intensity of fluorescent radiation of $\mathrm{Cr}$ and $\mathrm{Se}$ and the position of the $\mathrm{Zn}_{0.81} \mathrm{Cd}_{0.20} \mathrm{Cr}_{1.99} \mathrm{Se}_{4}$ crystal. Figure 3 presents also the $\mathrm{Cr}$ and Se concentration calculated by the standardless FP for each crystal position. It can be noticed that in a wide range
Table $3 \mu$-XRF analysis for the single crystal of $\mathrm{ZnCdSrSe} \mathrm{kept}$ in the same position and from various sides of the crystal

Results are in percent $(\mathrm{m} / \mathrm{m})$

\begin{tabular}{|c|c|c|c|c|c|c|c|c|}
\hline \multirow[t]{2}{*}{ No. of measurement } & \multicolumn{4}{|c|}{ The same position of the crystal } & \multicolumn{4}{|c|}{ Various sides of the crystal } \\
\hline & $\mathrm{Zn}$ & $\mathrm{Cd}$ & $\mathrm{Cr}$ & $\mathrm{Se}$ & $\mathrm{Zn}$ & $\mathrm{Cd}$ & $\mathrm{Cr}$ & $\mathrm{Se}$ \\
\hline 1 & 10.73 & 4.50 & 20.90 & 63.80 & 10.73 & 4.50 & 20.90 & 63.80 \\
\hline 2 & 10.79 & 4.38 & 20.77 & 64.00 & 10.83 & 4.40 & 20.89 & 63.84 \\
\hline 3 & 10.77 & 4.40 & 20.79 & 63.99 & 10.71 & 4.62 & 20.51 & 64.10 \\
\hline 4 & 10.76 & 4.38 & 20.95 & 63.84 & 10.72 & 4.52 & 20.62 & 64.10 \\
\hline 5 & 10.71 & 4.42 & 20.88 & 63.91 & 10.64 & 4.58 & 20.05 & 64.70 \\
\hline Mean & 10.75 & 4.41 & 20.86 & 63.91 & 10.73 & 4.52 & 20.59 & 64.11 \\
\hline SD & 0.03 & 0.05 & 0.08 & 0.09 & 0.07 & 0.09 & 0.35 & 0.36 \\
\hline RSD (\%) & 0.30 & 1.2 & 0.36 & 0.14 & 0.63 & 1.9 & 1.7 & 0.56 \\
\hline
\end{tabular}



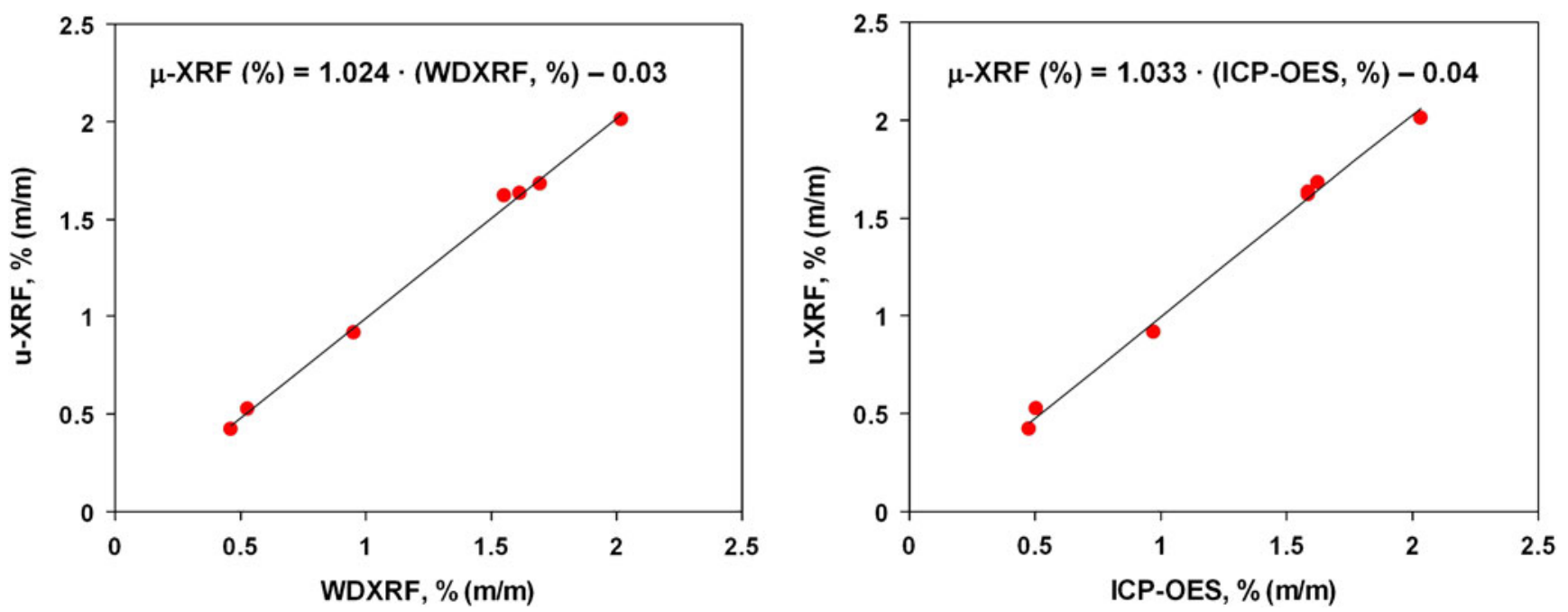

Fig. 4 The concentration of indium determined by $\mu$-XRF and WDXRF (ICP-OES) in seven crystals of $\mathrm{Cu}_{x} \mathrm{In}_{y} \mathrm{Cr}_{z} \mathrm{Se}_{4}$

of the sample positions the results are close to those obtained for the sample in the central position, even if the analyzed crystal is only partially irradiated by an X-ray beam. This can be explained by the fact that all elements in the crystal are quantified, and the results are normalized during the FP calculations. Table 2 presents the analysis for $\mathrm{Zn}_{0.81} \mathrm{Cd}_{0.20} \mathrm{Cr}_{1.99} \mathrm{Se}_{4}$ crystal of dimensions of $900 \mu \mathrm{m}$ adjusted in the central position and $\pm 600 \mu \mathrm{m}$ off-center, where the specimen is only partially excited by the primary $\mathrm{X}$-ray beam. In the off-center position, the fluorescent radiation is emitted not only from the front but also from the slanted faces of the crystal. Table 2 presents also the analysis performed with the $2,000-\mu \mathrm{m}$ collimator, which gives the $2,888-\mu \mathrm{m}$ focal spot size, thus larger than the crystal size. These results indicate that the best accuracy can be obtained if the crystal is precisely set in the central position.

Taking into account the effect of crystal position on the results of analysis, the precision under repeatability conditions was evaluated for several monocrystals. Table 3 gives exemplary analyses for $\mathrm{Zn}_{0.81} \mathrm{Cd}_{0.20} \mathrm{Cr}_{1.99} \mathrm{Se}_{4}$. The measurements were repeated for the same and for various positions of the crystal. The standard deviations (SD) calculated for various positions are two to four times higher than those calculated for the measurements carried out without moving the sample. In general, if the crystal is precisely positioned, the relative standard deviation (RSD) should not be higher than $2 \%$.

The results of $\mu$-XRF analysis obtained by using the standardless FP method were compared with those resulting from ICP-OES and WDXRF, requiring the sample digestion. For this purpose, the measurements were done for several crystals of $\mathrm{ZnCr}_{2} \mathrm{Se}_{4}, \mathrm{CdCr}_{2} \mathrm{Se}_{4}$, and $\mathrm{CuCr}_{2} \mathrm{Se}_{4}$ doped with $\mathrm{V}, \mathrm{Ga}$, In, and $\mathrm{Cd}$ [15-18]. The agreement between the results appears to be quite good, as the relative differences in the analyses do not exceed $5 \%$. Figure 4 shows the relationships between the indium concentration in seven samples of $\mathrm{Cu}_{x} \mathrm{In}_{y} \mathrm{Cr}_{z} \mathrm{Se}_{4}$ determined by $\mu$-XRF and WDXRF or ICP-OES. The mean relative difference between $\mu$-XRF and WDXRF is approximately 3\%, whereas between $\mu$-XRF and ICP-OES it amounts approximately to $4 \%$.
Table 4 ED-EPMA analysis for ZnCdSrSe single crystal taken from the same and various areas of the sample

Results are in percent $(\mathrm{m} / \mathrm{m})$

\begin{tabular}{|c|c|c|c|c|c|c|c|c|}
\hline \multirow[t]{2}{*}{ No. of measurement } & \multicolumn{4}{|c|}{ The same area of the sample $(50 \times 50 \mu \mathrm{m})$} & \multicolumn{4}{|c|}{ Various areas $(50 \times 50 \mu \mathrm{m})$} \\
\hline & $\mathrm{Zn}$ & $\mathrm{Cd}$ & $\mathrm{Cr}$ & $\mathrm{Se}$ & $\mathrm{Zn}$ & $\mathrm{Cd}$ & $\mathrm{Cr}$ & $\mathrm{Se}$ \\
\hline 1 & 10.26 & 5.09 & 20.38 & 64.27 & 11.42 & 4.95 & 20.01 & 63.62 \\
\hline 2 & 10.80 & 5.37 & 20.27 & 63.55 & 14.18 & 6.02 & 23.69 & 56.11 \\
\hline 3 & 12.61 & 5.17 & 19.95 & 62.27 & 11.87 & 4.42 & 19.54 & 64.16 \\
\hline 4 & 11.26 & 4.11 & 20.00 & 64.63 & 10.57 & 5.25 & 18.88 & 65.29 \\
\hline 5 & 10.18 & 4.86 & 20.17 & 64.79 & 10.26 & 5.09 & 20.38 & 64.27 \\
\hline Mean & 11.02 & 4.92 & 20.15 & 63.90 & 11.66 & 5.15 & 20.50 & 62.69 \\
\hline $\mathrm{SD}$ & 1.0 & 0.5 & 0.2 & 1.0 & 1.5 & 0.6 & 1.9 & 3.7 \\
\hline RSD (\%) & 9.0 & 9.9 & 0.9 & 1.6 & 13.3 & 11.3 & 9.1 & 5.9 \\
\hline
\end{tabular}


Table 5 Nondestructive $\mu$-XRF and ED-EPMA analysis of ZnInCrSe and CdGaCrSe single crystals compared with wet analysis (WDXRF and ICP-OES after sample digestion)

\begin{tabular}{|c|c|c|c|c|c|c|}
\hline \multirow[t]{2}{*}{ Compounds } & \multirow[t]{2}{*}{ Element } & \multicolumn{3}{|c|}{ Concentration, \% (m/m) } & \multicolumn{2}{|l|}{ Relative difference, $\%$} \\
\hline & & $\mu-X R F$ & ED-EPMA & Wet analysis & $\mu-X R F$ vs. Wet analysis & ED-EPMA vs. Wet analysis \\
\hline \multirow[t]{4}{*}{ ZnInCrSe } & $\mathrm{Zn}$ & $10.2 \pm 0.1$ & $10.3 \pm 0.8$ & $10.1 \pm 0.6$ & 1.0 & 2.0 \\
\hline & In & $8.3 \pm 0.1$ & $9.8 \pm 0.6$ & $8.7 \pm 0.4$ & -4.6 & 12.6 \\
\hline & $\mathrm{Cr}$ & $18.5 \pm 0.1$ & $18.3 \pm 0.5$ & $19.2 \pm 0.7$ & -3.6 & -4.7 \\
\hline & $\mathrm{Se}$ & $63.0 \pm 0.2$ & $61.7 \pm 0.6$ & $62 \pm 1.6$ & 1.6 & -0.5 \\
\hline \multirow[t]{4}{*}{$\mathrm{CdGaCrSe}$} & $\mathrm{Cd}$ & $20.1 \pm 0.3$ & $22.9 \pm 0.7$ & $20.7 \pm 0.9$ & -2.9 & 10.6 \\
\hline & $\mathrm{Ga}$ & $0.26 \pm 0.03$ & $0.9 \pm 0.4$ & $0.4 \pm 0.1$ & -35.0 & 125.0 \\
\hline & $\mathrm{Cr}$ & $20.3 \pm 0.2$ & $18.3 \pm 0.5$ & $20.8 \pm 0.8$ & -2.3 & -12.0 \\
\hline & $\mathrm{Se}$ & $59.3 \pm 0.2$ & $57.9 \pm 0.7$ & $58 \pm 2$ & 2.3 & -0.2 \\
\hline
\end{tabular}

The higher values of SD for various positions of the crystal shown in Table 3 may result not only from the low precision in the crystal positioning but also from the inhomogeneous surface of the specimens. The surface inhomogeneity in our samples was investigated by EDEPMA. To do so, five measurements were made in the same area $(50 \times 50 \mu \mathrm{m})$ of $\mathrm{Zn}_{0.81} \mathrm{Cd}_{0.20} \mathrm{Cr}_{1.99} \mathrm{Se}_{4}$ crystal and five other ones in various areas. The resulting analyses are collected in Table 4. They are rather repeatable for the data collected in the same area, whereas the measurements performed in various areas give dispersed results. The concentrations vary within the following ranges: 10.3$14.2 \%, 4.4-6.0 \%, 18.9-23.7$, and $56.1-65.3 \%$ for $\mathrm{Zn}, \mathrm{Cd}$, $\mathrm{Cr}$, and $\mathrm{Se}$, respectively. This high dispersion can be explained by an inhomogeneous distribution of the ions in the spinel and also by a rough surface of the sample. Taking into account the inhomogeneity of the surface, the subsequent ED-EPMA measurements were carried out for the larger area of $200 \times 300 \mu \mathrm{m}$. The exemplary results of $\mu$-XRF and ED-EPMA analyses for $\mathrm{ZnInCrSe}$ and $\mathrm{CdGaCrSe}$ crystals, together with those from wet analyses (mean value of WDXRF and ICP-OES), are given in
Table 5. In general, the relative differences between the nondestructive ED-EPMA and the wet analysis can reach $10 \%$. Moreover, the very high errors should be expected for the elements at concentrations below $1 \%(\mathrm{~m} / \mathrm{m})$. It results from a nearness to the detection and quantity limits obtained by ED-EPMA. In case of low concentration of the doped elements in selenide spinel, the $\mu-X R F$ technique can successfully be applied. Detection limits for $\mu$-XRF vary from $0.01 \%$ to $0.05 \%(\mathrm{~m} / \mathrm{m})$ depending on the element to be determined (experimental conditions: 400- $\mu \mathrm{m}$ pinhole collimator, $45 \mathrm{kV}, 1,000 \mu \mathrm{A}, 10-\mathrm{min}$ counting time).

Considering further possibility of the nondestructive characterization of the selenide spinel crystals, the XPS method was also applied. XPS gives information only from the outer surface of the sample (up to approximately $10 \mathrm{~nm}$ ); therefore, to evaluate the effect of the surface preparation on the results of analysis, two modes of measurements were checked. First, the chemical composition was analyzed based on the spectrum detected from the sample without previous surface cleaning (after about $1 \mathrm{~h}$ in vacuum, the first measurement was made and revealed at
Table 6 Nondestructive XPS, $\mu$-XRF, and ED-EPMA analysis of $\mathrm{ZnCdCrSe}$ single crystal compared with wet analysis (WDXRF and ICP-OES after sample digestion)

\begin{tabular}{lllllr}
\hline Element & XPS, without scrapping & XPS, with scrapping & $\mu$-XRF & ED-EPMA & Wet analysis \\
\hline \multicolumn{2}{l}{ Determined concentration, \% $(m / m)$} & & & & \\
Zn & $7.0 \pm 0.4$ & $11.4 \pm 0.3$ & $10.73 \pm 0.08$ & $12.1 \pm 0.9$ & $10.9 \pm 0.8$ \\
$\mathrm{Cd}$ & $2.4 \pm 0.5$ & $4.1 \pm 0.3$ & $4.5 \pm 0.1$ & $4.1 \pm 0.6$ & $4.4 \pm 0.5$ \\
$\mathrm{Cr}$ & $34.4 \pm 0.6$ & $22.1 \pm 0.4$ & $20.9 \pm 0.2$ & $20.4 \pm 0.6$ & $21.3 \pm 0.9$ \\
$\mathrm{Se}$ & $56.2 \pm 1.1$ & $62.4 \pm 0.7$ & $63.8 \pm 0.2$ & $63.4 \pm 0.9$ & $63 \pm 1.5$ \\
$\mathrm{Re}$ & & & & \\
$\mathrm{Zn}$ & -36 & 4.6 & -1.6 & 11.0 & \\
$\mathrm{Cd}$ & -45 & -6.8 & 2.3 & -6.8 & \\
$\mathrm{Cr}$ & 62 & 3.8 & -1.9 & -4.2 & \\
$\mathrm{Se}$ & -11 & -1.0 & 1.3 & 0.6 & \\
\hline
\end{tabular}


dirt surface, in atomic percents, $80.1 \% \mathrm{C}, 16.4 \% \mathrm{O}, 2.4 \%$ Se, $0.4 \% \mathrm{Cr}, 0.4 \% \mathrm{Zn}, 0.2 \% \mathrm{~N}$, and $0.1 \% \mathrm{Cd}$ ). Next, the sample with a fresh surface obtained by scraping in situ in $10^{-10}-\mathrm{hPa}$ vacuum was measured (that time the composition was $62.4 \% \mathrm{Se}, 22.1 \% \mathrm{Cr}, 11.4 \% \mathrm{Zn}$, and $4.1 \% \mathrm{Cd}$, the contamination, i.e., $\mathrm{C}, \mathrm{O}$, and $\mathrm{N}$, was less than $0.1 \%$ ). Table 6 shows the results for $\mathrm{Zn}_{0.81} \mathrm{Cd}_{0.20} \mathrm{Cr}_{1.99} \mathrm{Se}_{4}$ crystal obtained with and without surface scraping; for comparison consistency in calculations for dirt surface, we omit contamination $(\mathrm{C}, \mathrm{O}$, and $\mathrm{N})$. For comparison, the results of the $\mu$-XRF, ED-EPMA, and wet analysis are also given. It turned out that the reliable chemical composition can be obtained only if the measurement is made for the fresh surface prepared by scraping in situ in $10^{-10}-\mathrm{hPa}$ vacuum. The accuracy of $10 \%$ is typically quoted for atomic concentrations routinely determined from the XPS experiments. However, the quantitative chemical analysis relies on several factors; therefore, standard assumptions may result in enormous errors in quantification [19, 20]. Also, the contamination of the surface is a source of serious errors. The best method in obtaining a free of contamination fresh surface is cleaving or scraping the crystal in situ in ultrahigh vacuum. The ion etching, commonly used for surface cleaning, may cause preferential sputtering in multielemental samples that leads to a change in their chemical composition. Besides, the investigations show that not only the preferential sputtering takes place (Fig. 5) but also some amount of contamination still remains. Moreover, in effect of the etching, the crystal surface may be deteriorated. The case is illustrated in Fig. 6, where the splitting of the $2 p_{3 / 2}$ peak disappears after the sputtering. Moreover, a small broadening of the XPS bands is observed. The $2 p_{3 / 2}$ peak splitting is typical for $\mathrm{Cr}^{3+}$ and can be observed only in the high-resolution measurements of surface of good-quality single crystals. The nature of the splitting is described in the literature [3].

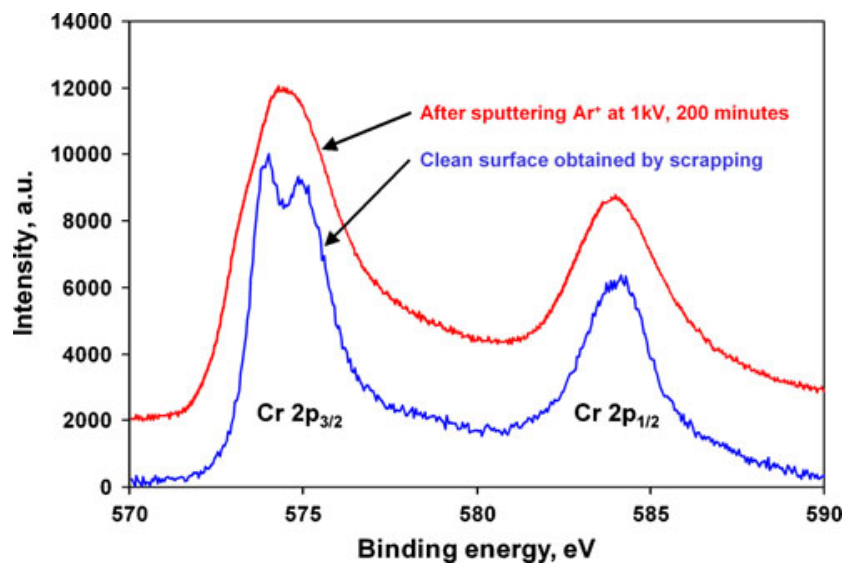

Fig. 6 Chromium $2 p$ core levels for the contamination-free crystal fresh surface obtained by scrapping in ultrahigh vacuum and after sputtering. The surface deterioration by sputtering reveals as a disappearance of the $2 p_{3 / 2}$ peak splitting

\section{Conclusion}

The present study shows satisfactory agreement between the results of the standardless $\mu$-XRF analysis made by using the fundamental parameters method and the results obtained with the techniques requiring sample digestion (WDXRF and ICP-OES analysis using reference samples). The best accuracy can be obtained if the measured single crystal is precisely positioned. Then the difference between $\mu$-XRF and wet analysis does not exceed 5\% rel. The reliable results of ED-EPMA can be obtained only if the measured area is sufficiently large, i.e., of $200 \times 300 \mu \mathrm{m}$. Even if this condition is fulfilled, the relative difference between the nondestructive ED-EPMA techniques and the wet analysis may reach $10 \%$. Moreover, the high errors should be expected in the determination of elements at the concentrations below $1 \%(\mathrm{~m} / \mathrm{m})$. In case of the XPS analysis, the accuracy of
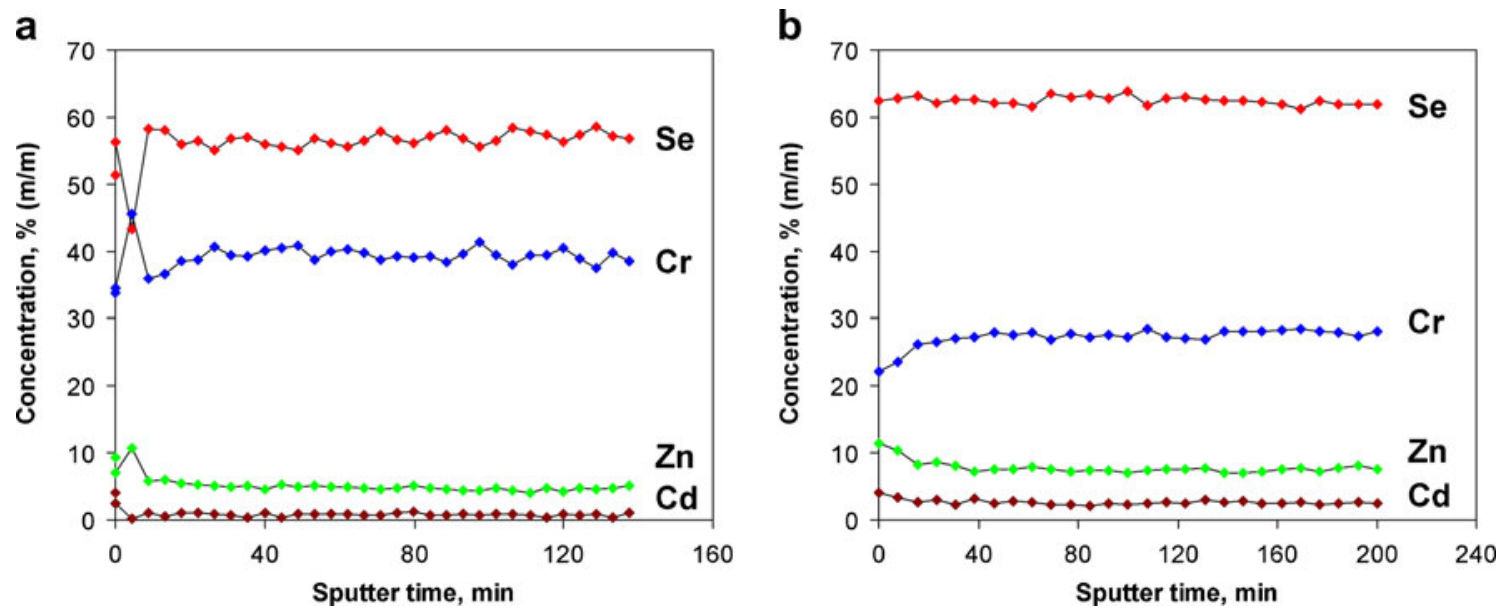

Fig. 5 Depth profiling analysis for the sample $(\mathrm{ZnCdCrSe})$ without previous surface cleaning (a) and after scrapping the surface (b) 
results depends on the proper preparation of the sample surface. It should be free of contamination that can be obtained by scraping in situ in ultrahigh vacuum. The ion etching, commonly used for cleaning the surface, leads to preferential sputtering; therefore, the reliable results cannot be obtained.

Acknowledgments The work was supported by the Ministry of Science and Higher Education by grant no. N N204 145938.

Open Access This article is distributed under the terms of the Creative Commons Attribution Noncommercial License which permits any noncommercial use, distribution, and reproduction in any medium, provided the original author(s) and source are credited.

\section{References}

1. Rudolf T, Kant Ch, Mayr F, Hemberger J, Tsurkan V, Loidl A (2007) Phys Rev B 76:174307-174310

2. Groń T, Mazur S, Duda H, Krok-Kowalski J, Malicka E (2007) Phys B 391:371-379

3. Malicka E, Waśkowska A, Heimann J, Mydlarz T, Sitko R, Kaczorowski D (2008) J Solid State Chem 181:1970-1976

4. Krok-Kowalski J, Warczewski J, Duda H, Gusin P, Krajewski K, Śliwińska T, Pacyna A, Mydlarz T, Malicka E, Kita A (2007) J Alloys Compd 430:39-42
5. Jurczyk J, Sitko R, Zawisza B, Buhl F, Malicka E (1999) Microchim Acta 132:41-47

6. Jurczyk J, Buhl F, Wilczek I (1993) Chem Anal (Warsaw) 38:519-526

7. Queralt I, Ibañez J, Marguí E, Pujol J (2010) Spectrochim Acta B 65:583-586

8. Baake O, Hoffmann PS, Flege S, Ortner HM, Gottschalk S, Berky W, Balogh AG, Ensinger W, Beckhoff B, Kolbe M, Gerlach M, Pollakowski B, Weser J, Ulm G, Haschke M, Blokhina E, Peter M, Porta D, Heck M (2009) Anal Bioanal Chem 393:623-634

9. Mino L, Agostino A, Codato S, Lamberti C (2010) J Anal At Spectrom 25:831-836

10. Sitko R, Zawisza B, Malicka E (2008) Spectrochim Acta B 63:1303-1308

11. Sitko R, Zawisza B, Malicka E (2009) Spectrochim Acta B 64:436-441

12. Sherman J (1955) Spectrochim Acta 7:283-306

13. Pella PA, Feng L, Small JA (1985) X-Ray Spectrom 14:125-135

14. Pella PA, Feng L, Small JA (1991) X-Ray Spectrom 20:109-110

15. Waśkowska A, Gerward L, Staun Olsen J, Morgenroth W, Malicka E, Skrzypek D (2008) J Phys Condens Mater 20:425209

16. Malicka E, Groń T, Pacyna AW, Maciążek E, Duda H, Pawełczyk M, Zawisza B, Sitko R (2009) J Alloys Compd 480:67-69

17. Malicka E, Waśkowska A, Skrzypek D, Sitko R, Kaczorowski D (2009) J Solid State Chem 182:3149-3154

18. Malicka E, Groń T, Skrzypek D, Pacyna AW, Badurski D, Waśkowska A, Mazur S, Sitko R (2010) Philos Mag 90:1525-1541

19. Tougaard S (1996) Appl Surf Sci 100/101:1-10

20. Tougaard S (2010) J Electron Spectrosc 178-179:128-153 